\title{
EYE TRACKING STUDY OF FRONTAL AND PROFILE FACE IMAGE OBSERVATION AND RECOGNITION
}

\author{
Andrej Iskra \\ University of Ljubljana, Faculty of Natural Sciences and Engineering, Department of Textiles, \\ Graphic Arts and Design, Chair of Information and Graphic Arts Technology, Ljubljana, Slovenia
}

\begin{abstract}
Facial images are an important element of nonverbal communication. Eye-tracking systems enable us to objectively measure and analyse the way we look at facial images and thus to study the behaviour of observers. Different ways of looking at facial images influence the process of remembering faces and recognition performance. In the real world we are dealing with different representations of faces, especially when we look at them from different angles. Memory and recognition performance are different when test subjects look at the face from the frontal or from a profile view. We studied crossobservation and recognition, so we performed two tests. In the first test, subjects observed facial images shown in the frontal view and recognized them in the profile view. In the second test, the faces were observed from the profile and recognized in the frontal view. The presentation time in the observation test was four seconds, which was found to be an adequate time for sufficient recognition in some previous tests. The results were analysed with the well-known time and spatial method based on fixations and saccades and with the new area method using heatmaps of the eye tracking results. We found that the recognition success (correct and incorrect recognition) was better when the combination of frontal view and profile recognition was used. The results were then confirmed by measuring the fixation duration and saccade length. More visible facial features resulted in a shorter fixation duration and shorter saccade length, which led to a better memory. We also confirmed the results of observation and recognition by area analysis, where we measured the area, perimeter and circularity of heatmaps. Here we found that larger areas and perimeter and smaller circularity of heatmaps resulted in better memory of facial images and therefore better recognition.
\end{abstract}

Key words: Face image, eye tracking, facial features, face recognition, fixation time

\section{INTRODUCTION}

We see faces in nature when we communicate with other people in different representations. We talk about different angles in the representation of facial images and also about the representation of faces in different emotional states. In our study, we limited ourselves to a neutral facial expression and defined two different angles of representation of the facial image (frontal and profile representation). Some previous studies (Brielmann et al, 2014) have shown a better memory for facial images in frontal view. There the researchers used the same representations in the recognition test. However, in our research we combined different representations of facial images in the observation and recognition process. We were interested in which combination of observation and recognition of facial images was better: observation of the frontal view and recognition of the profile representation or the inverse combination of observation of the profile representation and recognition of the frontal view. Researchers typically measure the fixations duration and the saccades length from which they can determine the appearance and thus the memory performance of facial images (Hsiao and Cottrel, 2008). In our study, we also used a relatively new method of heatmap analysis, in which we measure the area, perimeter and circularity of the viewing areas (Iskra, 2020). To obtain these results, we used eye tracking technology, which is considered the most objective method in this field and has recently been widely used in cognitive studies, ergonomics, psychology, marketing, security, etc. (Senior and Bolle, 2002).

\section{METHODS}

\subsection{Participants}

Our tests were attended by 22 test participants, 6 male and 17 female (average age of 20.6, SD = 1.02). They were our students, all of whom had normal vision. The participants received a bonus in the evaluation of their study. 


\subsection{Stimuli}

For testing purposes, we took 40 male and 40 female facial images from the Minear and Park database (Minear and Park, 2000). We selected 20 faces for both genders, as well as a frontal and a profile image for each face. An example of two face images (male frontal view and female profile view) is shown in Figure 1. The dimensions of the facial images were $800 \times 800$ and were displayed at a distance of $60 \mathrm{~cm}$ from the screen in a size corresponding to the conditions of natural observation (face viewed at a distance of 1 meter) (Henderson et al, 2005).

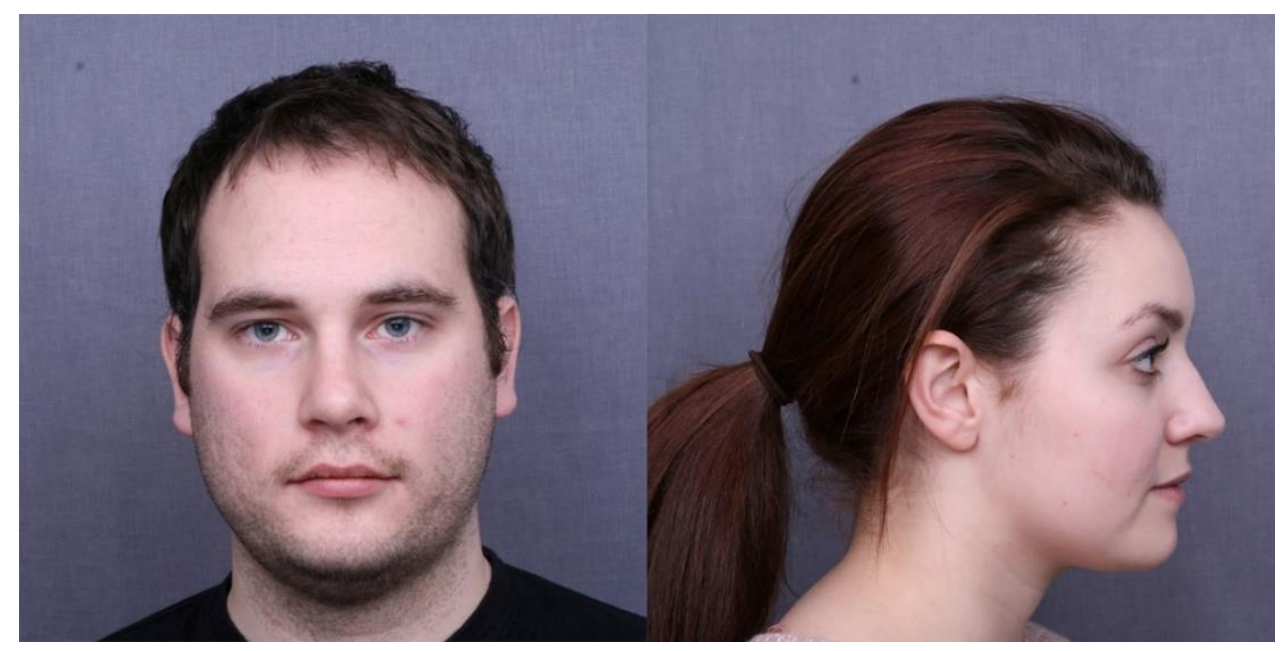

Figure 1: Stimuli facial images

\subsection{Apparatus}

The tests were carried out in the Laboratory of Visual Perception and Colorimetry at the Department of Textile, Graphic and Design of the Faculty of Natural Sciences and Engineering at the University of Ljubljana. When setting up the environmental and testing system we followed the standards and recommendations (Pernice and Nielsen, 2009).

We performed the test with the Tobii X-120 eye tracking system. The distance between the test subjects and the screen with the facial images was $60 \mathrm{~cm}$. The setting of the test environment and the test subject is shown in Figure 2.

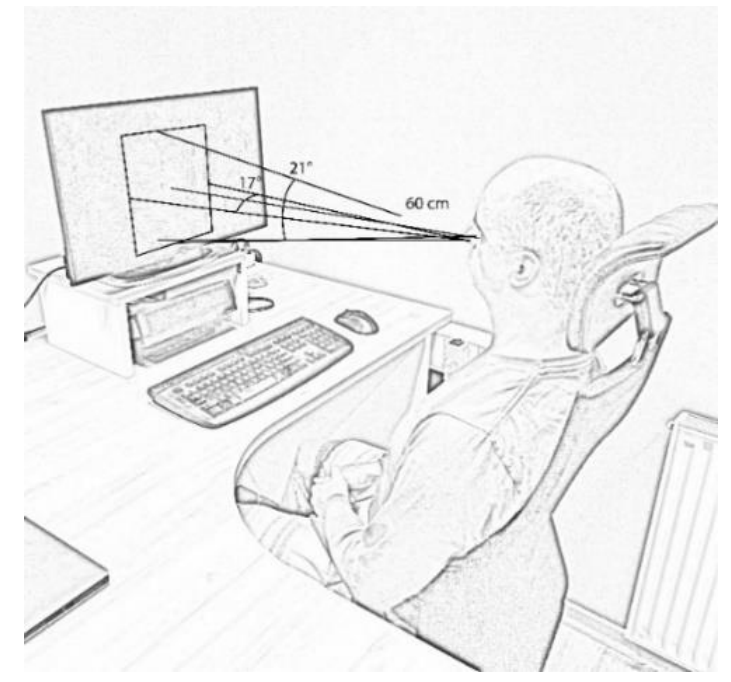

Figure 2: Testing setup

Analysis were done in Tobii Studio 3.4.8 software. The defaults setting for definition of fixation was 100 $\mathrm{ms}$ for 30 pixel area. That means if eyes stayed in the area 30 pixel for at least $100 \mathrm{~ms}$ it was concerned as one fixation (Tobii Studio, 2016). 


\subsection{Procedure}

We had two main tests, and both were divided into observation and recognition test. This is commonly referred as a memory test. However, cross-recognition meant that the images from the observation test and the recognition test were displayed at different angles. First test included 20 images of the frontal view (observation test) and 40 images of the profile view (recognition test), while second test had 20 images of the profile view in the observation test and 40 images of the frontal view in the recognition test. In this way, we wanted to find out which combination was more successful in remembering and later recognizing facial images. The observation test (Figure 3) was automatic, so that the test participants had no influence on the display of facial images which were presented for 4 seconds, a time that allows a satisfactory memory of facial images (Iskra, 2020). After the instructions and a black screen appeared to neutralize the participants gaze, then a facial image display followed for 4 seconds, then again a black screen for 2 seconds and a new facial image. The display was evenly distributed between male and female facial images.

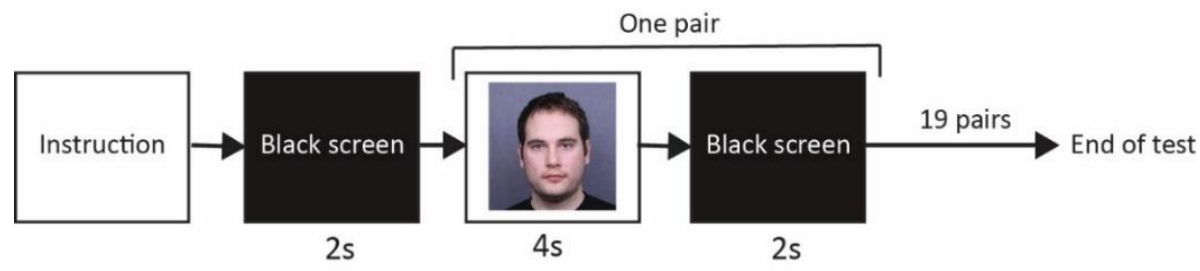

Figure 3: Procedure of observation test

However, the recognition test was controlled by the participants. After the instructions and a 2 second black screen, the facial image was displayed in a different view than in the observation test. When the participants answered whether or not they had seen the face in the observation test, they clicked the mouse button and a new facial image appeared. Recognition test contained 40 facial images. The procedure is shown in Figure 4.

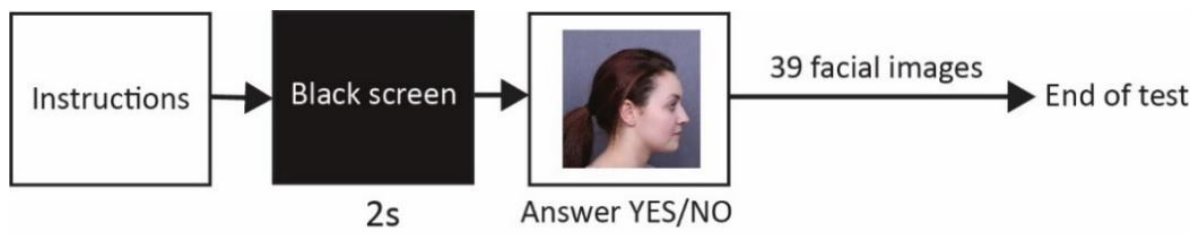

Figure 4: Procedure of recognition test

\subsection{Analysis of results}

We presented the results in several ways. The recognition results were presented as correct and incorrect recognition. The correct recognition means that the facial image was the observation test, and the test participant confirmed that in the recognition test. However, incorrect recognition, means that for a particular facial image in the recognition test, the participants answered that they had seen it in the observation test, but it was not really there.

Another group of results was measuring the fixation duration and saccade length. The fixation duration was obtained directly from Tobii Studio, and saccade length was calculated by as a distance between two consecutive fixations, as shown in Equation 1.

$\mathrm{F}_{1} \mathrm{~F}_{2}=\sqrt{\left(\mathrm{x}_{1}-\mathrm{x}_{2}\right)^{2}+\left(\mathrm{y}_{1}-\mathrm{y}_{2}\right)^{2}}$

The saccade length then had to be converted from px to angular degrees ${ }^{\circ}$ using Equation 2.

$\alpha=\arctan \frac{F_{1} F_{2}}{60 R}$

where $\mathrm{R}$ is the screen resolution. In our case we set $27.5 \mathrm{px} / \mathrm{cm}$.

However, the third group of results was the heatmap analysis, where we analysed their area, perimeter and circularity. The procedure was as follows: colour heatmaps were converted to grayscale (in Tobii 
Studio) and grayscale were then converted to black and white in ImageJ. In this program, we also calculated the parameters of these heatmaps described above. The procedure is shown in Figure 5.
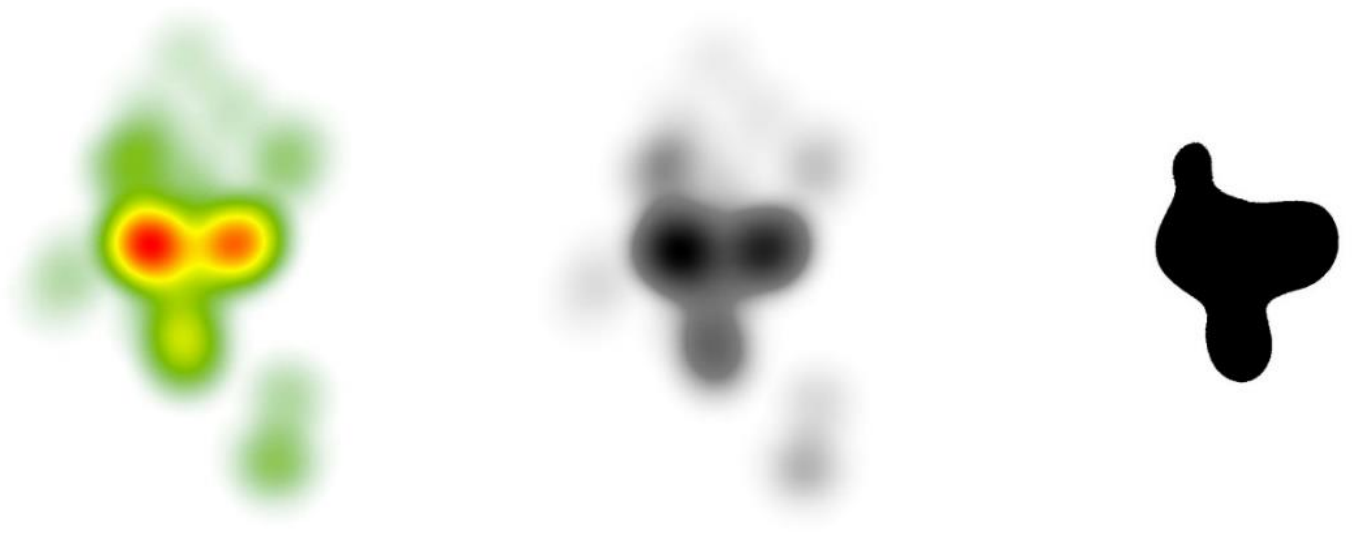

Figure 5: Colour, grey and BW heatmap

\section{RESULTS}

We compared the test results of two different conditions (frontal observation, profile recognition and opposite). Table 1 shows the correct and the incorrect recognition.

Table 1: Results of correct and incorrect recognition for both test combinations

\begin{tabular}{|l|c|c|}
\hline Test combination & Correct recognition & $\begin{array}{c}\text { Incorrect } \\
\text { recognition }\end{array}$ \\
\hline Frontal observation, profile recognition & $72,9 \%$ & $10,7 \%$ \\
\hline Profile observation, frontal recognition & $69,2 \%$ & $25,4 \%$ \\
\hline
\end{tabular}

Further results were fixation duration and saccade length (Table 2). In the controlled observation tests (4second image display) they were more comparable than in the recognition test, in which the image display times were controlled by the participants themselves when they made decision of the displayed facial image. These times were different for each facial image for each participant.

Table 2: Results of fixation duration in saccade length for both test combinations

\begin{tabular}{|l|c|c|c|c|}
\hline \multirow{2}{*}{ Test combination } & \multicolumn{2}{|c|}{ Fixation duration (ms) } & \multicolumn{2}{c|}{ Saccade length $\left(^{\circ}\right)$} \\
\cline { 2 - 5 } & Observation & Recognition & Observation & Recognition \\
\hline Frontal observation, profile recognition & 320 & 308 & 3,79 & 4,97 \\
\hline Profile observation, frontal recognition & 349 & 266 & 4,72 & 4,14 \\
\hline
\end{tabular}

We analysed heatmaps for both cross-tests, but only for the observation process, since we had controlled conditions (observation time 4 seconds). The results of the area, perimeter and circularity of heatmaps and are shown in Table 3.

Table 3: Results area, perimeter and circularity of heatmaps in the observation test

\begin{tabular}{|l|c|c|c|}
\hline Test combination & Area $(\mathrm{px})$ & Perimeter $(\mathrm{px})$ & circularity \\
\hline Frontal facial images & 46029 & 888 & 0,739 \\
\hline Profile facial images & 39269 & 784 & 0,805 \\
\hline
\end{tabular}




\section{DISCUSSION}

As can be seen from Table 1, the correct recognition was better for the combination of frontal view observation and profile recognition than the reverse combination. An even greater difference occurs with incorrect recognition. The reason is the poorer memory of profile facial images, because in profile view there are fewer facial features according to which we remember and distinguish faces. With correct identification, this difference is insignificant (72.9\% vs. 69.2\%), but with incorrect recognition a greater difference occurs (25.4\% vs. $10.7 \%$ ). Similar results of significantly worse identification when observing profile face images compared to frontal facial images were also found by other researchers (Iskra, 2020). Regarding fixation duration, we see that the fixation duration was shorter in the observation for frontal facial images. This can be seen in both the observation test (frontal $320 \mathrm{~ms}$, profile $349 \mathrm{~ms}$ ) and the recognition test (frontal $266 \mathrm{~ms}$, profile $308 \mathrm{~ms}$ ). We see the reason for this in the greater number of facial features in frontal view that attracted participants gaze, so that there are more eye movements and the fixations are consequently shorter. Here the results are more relevant for the observation process, because we had controlled conditions with the same observation time for all participants and for all facial images (4 seconds) and in this test the eyes calmed down more and looked at the facial images more relaxed (longer fixations). During recognition, the participants controlled facial image display and the test was faster, so the eyes were more "active" and the fixation duration was shorter. However, the results of the fixation duration in the observation process are a confirmation of the previous research (Iskra, 2020). The only difference is that a slightly longer fixation duration was found there (frontal facial images 331 $\mathrm{ms}$, profile facial images $362 \mathrm{~ms}$ ), but the difference is almost the same. The longer fixation duration in their research is due to the smaller facial image dimension, because with the smaller dimensions of facial images the fixation duration increases (Iskra, 2020).

Results of saccade lengths in the observation process shows that these were shorter in frontal facial images $\left(3.79^{\circ}\right)$ than in profile facial images $\left(4.72^{\circ}\right)$. Frontal facial images have more facial features that are relatively close together (eyes, nose, mouth), so saccades are shorter than in profile facial images, where fewer facial features are shown and are further apart (especially the ear is far away from an eye and the nose). Similarly, the saccades in the recognition test are shorter $\left(4.14^{\circ}\right)$ in frontal facial images than in profile facial images $\left(4.97^{\circ}\right)$. However, the saccades in the recognition process are usually longer than in the observation. We also performed a heatmap analysis. We did this for both cross-tests, but only for the observation process, because we had controlled conditions (observation time 4 seconds). The heatmaps were analysed by ImageJ, where we obtained area, perimeter and circularity. The results for the both facial images (frontal and profile) are shown in Table 3, and we can see that the area and perimeter of the heatmap are larger in the frontal facial images (46029 px and 888 px) than in the profile facial images (39269 px and 784 px). In the case of circularity, the result is the opposite, profile images have a higher circularity (0.805) than frontal images (0.739). These results can again be explained by the structure of the frontal and profile facial images themselves. Frontal facial images show several main facial features (eyes, nose, mouth), which are arranged further apart. In profile facial images, one eye and the nose are the main facial features that attract attention and are close together. Therefor the area of the heatmaps is smaller and consequently the perimeter is smaller. However, these areas are geometrically rounder and the circularity is greater. These results are confirmed by Figure 6, which shows the colour and black and white heatmap for frontal and profile facial images. In previous studies (Iskra, 2020), researchers have already shown that a larger area and perimeter, as well as a smaller circularity, leads to better memory for facial images, which was also confirmed in our results.

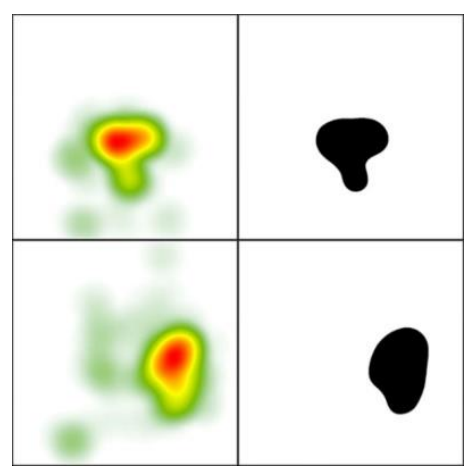

Figure 6: Colour and BW heatmap for frontal (top) and profile (bottom) facial images 


\section{CONCLUSIONS}

In our research we were interested in which combination of observation and recognition of facial images is better: observation of the frontal view and recognition of the profile view or the reverse combination of observation of the profile view and recognition of the front view. Even before the research, we predicted better results in frontal facial image observation and profile recognition. The reason is that frontal facial image contains more facial features, so the face provides more information that helps us to remember it. This was also confirmed by the results, especially in case of incorrect recognition. The way we see and remember faces is also determined by the way facial image are displayed. In the frontal facial images, we see a larger part of the face, which was confirmed by the shorter fixation duration. There were more of these fixations, which in turn leads to a better memory of the facial image. A better memory for the frontal facial images due to the placement of the facial features was also confirmed by shorter saccade lengths. The results were also presented using a newly introduced method of heatmap analysis, where we confirmed that the memory of facial images is better in frontal views, where the area and perimeter of heatmaps are larger and circularity smaller.

\section{REFERENCES}

[1] Brielmann, A.A., Büthoff, I., Armann, R.: "Looking at faces from different angles: Europeans fixate different features in Asian and Caucasian faces", Vision Research 100, 105-112, 2014. doi: 10.1016/j.visres.2014.04.011.

[2] Henderson, J.M., Williams, C.C., Falk. R.J.: "Eye movements are functional during face learning", Memory \& Cognition 33, 98-106, 2005. doi: 10.3758/bf03195300.

[3] Hsiao, J.H., Cottrel, G.W. "Two fixations suffice in face recognition", Psychological Science 9, 9981006, 2008. doi: 10.1111/j.1467-9280.2008.02191.x.

[4] Iskra, A.: "Development of combined method for analysis of facial images using eye tracking system", PhD thesis, University of Ljubljana, 2020.

[5] Minear, M., Park, D.: "A lifespan database of adult facial stimuli", Behavior Research Methods, Instruments \& Computers 36, 360-363, 2000. doi: 10.3758/BF03206543.

[6] Pernice, K., Nielsen, J.: "Eyetracking methodology: how to conduct and evaluate usability studies using eyetracking", (Fremont, USA, Nielsen Norman Group, 2009.), page 164.

[7] Senior A.W., Bolle, R.: "Face recognition and its applications. Biometric Solutions for Authentication in an E-World", (Boston, USA, Kluwer Academic Publishers, 2002.), pages 101-115.

[8] User's Manual Tobii Studio version 3.4.5 (Tobii Technology, Danderyd, Sweden, 2016.), page 40.

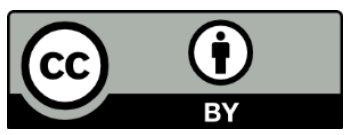

(C) 2020 Authors. Published by the University of Novi Sad, Faculty of Technical Sciences, Department of Graphic Engineering and Design. This article is an open access article distributed under the terms and conditions of the Creative Commons Attribution license 3.0 Serbia (http://creativecommons.org/licenses/by/3.0/rs/). 